\begin{tabular}{|c|c|c|}
\hline & $\begin{array}{l}\text { European Association for the } \\
\text { Development of Renewable Energies, Environment } \\
\text { and Power Quality (EA4EPQ) }\end{array}$ & $\begin{array}{l}\text { International Conference on Renewable Energies and Power Quality } \\
\text { (ICREPQ'12) } \\
\text { Santiago de Compostela (Spain), 28th to 30th March, } 2012\end{array}$ \\
\hline
\end{tabular}

\title{
Limiting Ramp Rate of Wind Power Output using a Battery Based on the Variance Gamma Process
}

\author{
D. Lee ${ }^{1}$ and R. Baldick ${ }^{1}$ \\ ${ }^{1}$ Department of Electrical Engineering \\ University of Texas at Austin \\ 2501 Speedway, Austin, TX 78712 (USA) \\ Phone/Fax number: +1 512 3504704, e-mail: Hello.Keanu@utexas.edu, baldick@ece.utexas.edu
}

\begin{abstract}
A battery system is designed to reduce violations of ramping limit due to severe and long ramps of wind power output. The power rating, battery capacity, and operational policies of the battery are decided based on the assumption that the wind power output follows the Variance Gamma (V.G.) process over short time interval. The histogram of the ramp rates of wind power follows the symmetric Laplace distribution. Furthermore, ramp rates have independent and stationary increments, and their variances are proportional to the time interval. Consequently, the wind power output can be modelled as a simple form of the V.G. process. Battery operational oplicies based on the V.G. process are tested using statistics representing the sum of all wind power outputs from wind farms in the Electric Reliability Council of Texas (ERCOT). It is found that the battery can essentially eliminate violations of ramping limits due to severe ramp events. Furthermore, in this operation, it is discovered that the power rating influences the ramp limiting performance of the battery more than battery storage rating.
\end{abstract}

\section{Key words}

Wind power, Ramp rate control, Battery, Laplace distribution, Variance Gamma process, Curtailment

\section{Introduction}

The ramp rate of wind power output is defined in [1] as the power changes from minute to minute, so its unit is [MW/minute]. Some ramp events result in severe ramp rates of power generation that exceed a "ramp rate limit" (RRL), which represents the capability of the remaining power system to compensate for wind ramps. Those ramp events, such as the sudden die-off and rise, not only disturb the balance of demand and supply, but also hamper the participation of wind power in the electricity market [2].

Wind power curtailment and reserve services can reduce severe ramp events, but they waste potential wind power energy and increase the thermal generation costs, respectively. Recently, a battery has been used to limit severe ramp events [3]. A battery can be charged during ramp-up events and discharged during ramp-down events without either wasting energy (except for energy loss in round trip charging and discharging of the battery) or requiring reserve services. The use of batteries can also help stakeholders maximize profits by arbitraging electricity prices. However, the power rating, battery capacity, and operation policies must be designed before establishing a battery system. Therefore, the goal of this paper is to design battery parameters in order to compensate for almost all severe ramp events of wind power output. However, in the pursuit of this goal, it will be helpful to address the hidden uncertainty driving wind power output and ramp events. Specifically, this paper assumes that the wind power output follows the simple form of the V.G. process with zero drift, zero mean, and zero skewness over a short time intervals of the order of 20 seconds. We show that this hypothesis is satisfied by the sum of all power outputs of wind farms in ERCOT.

We demonstrated that the ramp rates distribution follows the Laplace distribution, and showed that nonoverlapping ramp events are independent and identically distributed. We also showed that the variances of ramp rates are approximately linearly proportional to time differences. Based on these characteristics, battery parameters and operation policies are designed. The organization of this paper is as follows. In section II, the V.G. process is introduced, and it is shown that the wind power output follows the V.G. process. Section III explains the battery mechanism and decides battery parameters and operation policies. Section IV provides the simulation and its results. Section V is the conclusion.

\section{Variance-Gamma Process}

The V.G. process was originally proposed to address the observation that the increments of the logarithm of stock prices are distributed with a longer tail than the normal distribution. Since the distribution of the increment of the V.G. process generally follows the Laplace distribution, it is also called a generalization of the Laplace process. To include these characteristics, the V.G. process is 
defined as the random time-changed Brownian Motion (B.M.), with the time change of the B.M. following an independent and identically distributed gamma distribution. The B.M., whose increments follow the normal distribution, and the Laplace motion are special cases of the V.G. process [4].

There are four conditions under which the given time series will follow the V.G. process. The first condition is that the increment of the time-series at the given time interval should follow the probability density function (PDF) of the V.G. process. The PDF is defined in [5] as

$$
\begin{aligned}
f_{X}(x)= & \frac{\left(\alpha^{2}-\beta^{2}\right)^{\lambda}}{\sqrt{\pi} \Gamma(\lambda)(2 \alpha)^{\lambda-0.5}}|x-\mu|^{\lambda-0.5} \\
& \times K_{\lambda-0.5}(\alpha|x-\mu|) \exp (\beta(x-\mu))
\end{aligned}
$$

where $K_{\lambda-0.5}(\mathrm{z})$ is the Bessel function of the second kind of order $\lambda-0.5$, and where $\Gamma(\lambda)$ is the gamma function. The parameter $\alpha$ specifies the kurtosis, $\beta$ specifies the skewness, $\mu$ specifies the mean, while $\lambda$ specifies the shape of the distribution. The second condition is that the timeseries $X$ should start at the origin, i.e., $X(0)=0$. The third condition is to have independent and stationary increments [6]. The final condition is that the variances are proportional to the time intervals.

To show that the wind power output follows the V.G. process, the total wind power from all wind farms in the ERCOT sampled at every four seconds in May 2008 is used. Four seconds data is averaged using twenty seconds moving average to generate 20 seconds sampled power data. The total capacity of all wind farms in ERCOT in this month is around $8000 \mathrm{MW}$, and the maximum wind power in May 2008 was 4000 MW. The histogram of the ramp rate of wind power is shown in Fig. 1.

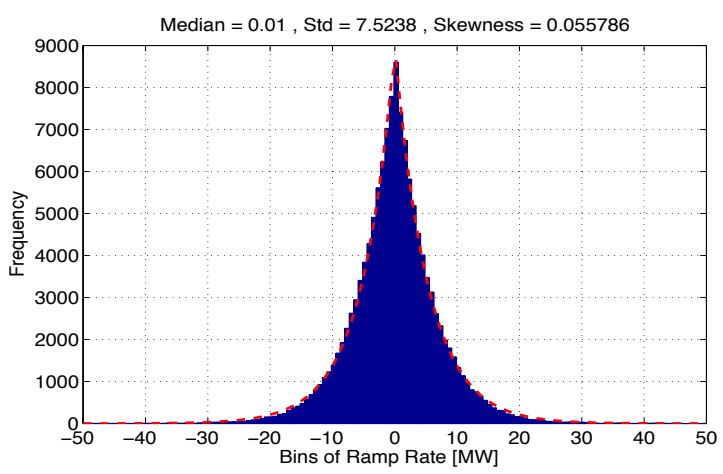

Fig. 1. The distribution of the ramp rate follows the symmetric Laplace distribution. The red dotted line represents the symmetric Laplace distribution.

The distribution in Fig. 1. follows the symmetric Laplace distribution with zero mean as mentioned in [5], and this phenomenon is also supported by the statistics in [7]. Wind power outputs sampled on other months also follow the Laplace distribution. To see that the distribution in Fig. 1. follows the PDF in (1), parameters in (1) are found to correspond to a symmetric Laplace distribution with the same standard deviation in Fig. 1. First, since the distribution in Fig. 1 is symmetric and has zero mean, both $\beta$ and $\mu$ are zero. Therefore, the PDF in (1) becomes

$$
f_{X}(x)=\frac{\alpha^{2 \lambda}|x|^{\lambda-0.5}}{\sqrt{\pi} \Gamma(\lambda)(2 \alpha)^{\lambda-0.5}} K_{\lambda-0.5}(\alpha|x|)
$$

However, since the histogram in Fig. 1. closely follows the Laplace distribution, the PDF of the Laplace distribution is used to find $\alpha$ and $\lambda$. The Laplace PDF is defined in [4] as

$$
f_{X}(x)=\frac{1}{\sqrt{2} \sigma} e^{\frac{-\sqrt{2}|x|}{\sigma}}
$$

where $\sigma$ is the standard deviation, which is 7.5238 for the histogram in Fig. 1. In order to match (1) to (3), note that the Bessel function $K_{0.5}(\alpha \mathrm{x})$ can be approximated as

$$
K_{0.5}(\alpha x) \approx \sqrt{\frac{\pi}{2 \alpha x}} e^{-(\alpha x)}
$$

Consequently, $\lambda=1$. By comparing (2) and (3), the parameter $\alpha$ becomes:

$$
\alpha=\frac{\sqrt{2}}{\sigma}
$$

Summarising, all parameters in (1) are specified in Table I .

Table I. Parameters in the V.G. process

\begin{tabular}{|c|c|c|c|}
\hline$\alpha$ & $\beta$ & $\mu$ & $\lambda$ \\
\hline 0.115 & 0 & 0 & 1 \\
\hline
\end{tabular}

The second condition is satisfied because our focus on ramp rates means that we can assume that wind power output always starts from zero. The third condition is also verified in Fig. 2. which shows the distribution of ramp rates on the condition that the previous ramp event was a ramp-up. The distribution is a little skewed and is shifted to the right. However, it essentially follows the same Laplace distribution as the unconditioned ramps having a similar standard deviation. Therefore, it could be concluded that increments are independent and stationary over very short time interval.

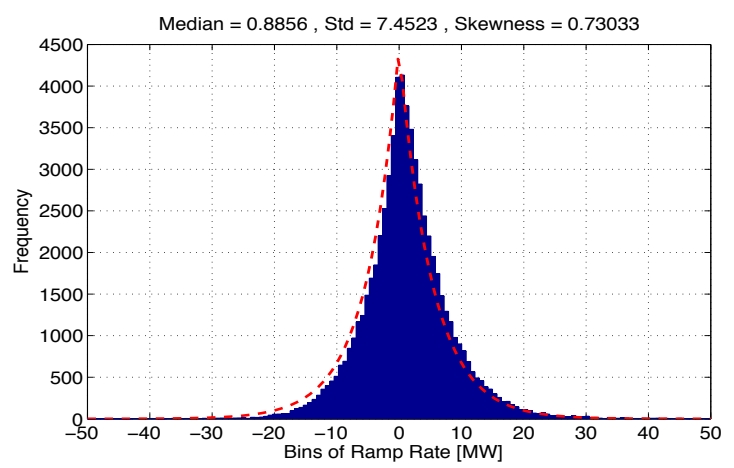

Fig. 2. The conditional distribution of the ramp rate after a ramp-up event is a skewed a little, and it is shifted to the right. 
Fig. 3. Verifies that the final condition holds approximately. It shows that the change of the variance is linearly proportional to the duration of the time interval over which the ramp is calculated. However, variances become a quadratic function of the duration of the time interval as the duration increases, so the wind power output follows the V.G. process only for short time intervals. Seasonal trends, diurnal periods, or chaotic meteorology could be the reason for this deviation over longer periods.

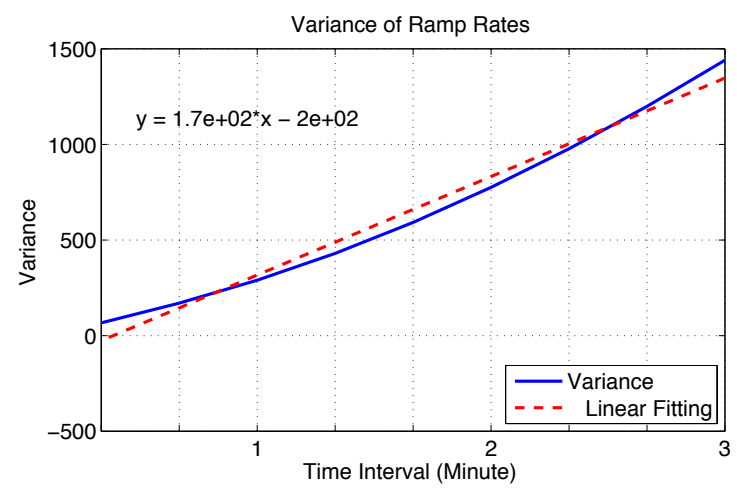

Fig. 3. Changes of variance is approximately linearly proportional to duration of the time intervals, over which the ramp is calculated for duration up to three minutes.

To summarize, wind power output can be modelled as the V.G. process within a three-minute time period.

\section{Battery Operation}

In this section, the battery mechanism is introduced, and then operating policies and battery parameters, such as a power rating and battery capacity, are designed based on the assumption that the wind power output follows the simple form of the V.G. process.

Basically, a battery device can limit severe ramp events by increasing charge power when there is a ramp-up event, and by increasing discharge power when there is a rampdown event. Contrary to conventional generators, a battery does not have significant ramp constraints. However, it is limited by the power rating, which represents the amount of exchangeable power available at every operation time, and by the battery capacity, which represents a maximum exchangeable energy that can be stored. Furthermore, since the cost of a battery depends mostly on battery parameters, deciding the economic battery parameters should be designed from an economic perspective.

\section{A. Battery Mechanism}

There are three constraints on battery operation. First, the battery cannot be fully charged because of its internal resistance [3], so it is assumed that a battery can be charged only up to $90 \%$ of its capacity, and it can be discharged only down to $10 \%$ of its capacity. Therefore, $80 \%$ of the battery capacity is used. In addition, the level of charged energy with respect to the battery capacity is called the State of charge (SOC). Second, there is some round trip power loss between charging and discharging. To account for round trip losses, it is assumed that the battery loses $10 \%$ of the power when it charges. We adopt the convention that the battery power is negative when it charges, and that it is positive when it discharges. The overall structure of battery charging is explained in Fig. 4.

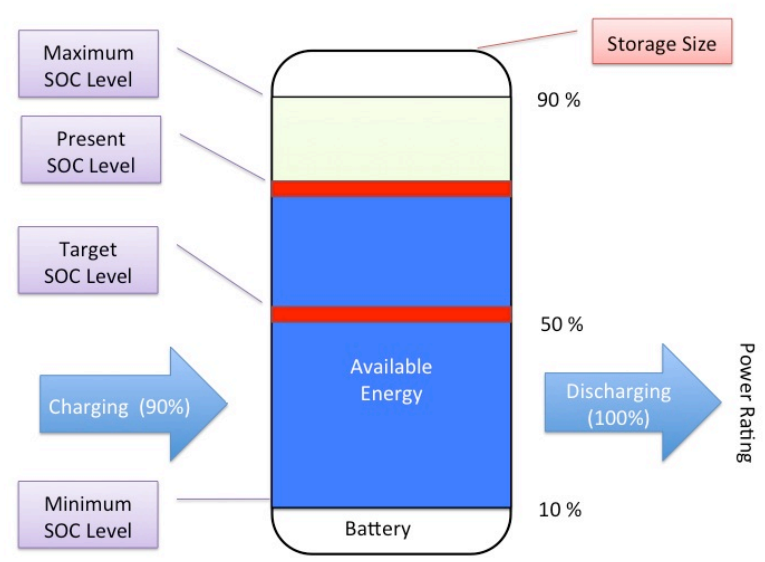

Fig. 4. The analysis of battery is displayed. Energy can only be stored between the maximum and minimum SOC levels. It is assumed that there is a $10 \%$ energy loss when it charges.

Third, at the beginning of every operation interval of length 20 seconds, the operation mode of the battery is updated. For every operation interval, the battery is in one of three conditions: charging, discharging, or inactive. It should be noted that the sampling interval of the time series and the operation interval of the battery are both 20 seconds.

Finally, important terminologies are introduced. The total output to the grid is the sum of battery output and wind power output. The net power output from wind farms and battery is called the net production, and the ramp rates of the net production should be minimized through control of the battery. The battery input is defined as the difference between the wind power and net production.

\section{B. Operation Policies}

In this subsection, three operation policies are introduced. Three policies are based on the characteristics of ramp rates mentioned above, and they decide the operation mode of a battery every 20 seconds.

The first strategy is to limit the ramp rates of net production within the RRL [3]. For example, when a ramp-up event happens, the battery charges within its power and maximum SOC limit. On the other hand, when a ramp-down event happens, the battery discharges power within its power and minimum SOC limit.

The second strategy is applied when the absolute values of ramp rates are within the RRL, and the SOC level is bigger than 0.55 or lower than 0.45 , A battery should be able to respond to both ramp events. 
For a ramp-up event:

1) When the SOC level is larger than 0.55 , the battery discharges as much power as possible without violating the upper RRL of the net production.

2) While, when the SOC level is lower than 0.45 , the battery charges as much power as possible without violating the lower RRL of the net production.

On the contrary, for a ramp-down event:

1) When the SOC level is higher than 0.55 , a battery discharges as much power as possible without violating the upper RRL.

2) While, when the SOC level is lower than 0.45 , the battery charges as much power as possible without violating the lower RRL.

The third strategy is applied when the absolute values of ramp rates are within the RRL, and the SOC level is between 0.45 and 0.55 . In this condition, the net production is allowed to follow the wind power. In other words, the battery neither charges nor discharges. The reason for this policies is that if the battery charges or discharges, it could increase the shock of the next ramp events. Therefore, following the wind power can reduce the ramp rates. All these policies should work within the maximum and minimum SOC level in Fig. 4.

\section{Battery Parameters Calculation}

The battery parameters are calculated recursively. In a longer time interval, consecutive same ramp events make larger ramp events. The frequency of larger ramp events differs from every wind power data, so they should be analyzed empirically, and battery parameters should also be estimated empirically. Furthermore, since battery inputs are affected by battery parameters, battery parameters should be estimated by increasing a small step from initial values and running the simulation repeatedly.

The first step is that the RRL, the level of ramp rates that a battery will satisfy, should be given. Furthermore, the satisfaction level (SL) should also be given. The SL is defined as the percentage between the time duration of ramp events whose power is compensated so that the ramp is within the RRL and duration of wind power data. There are three SL: the target SL to satisfy, original SL without a battery, and the compensated SL with a battery.

Second, the initial values of power rating and battery capacity are set based on the ramp rate distribution. The power rating and battery capacity will be increased from initial values until they satisfy the SL. The initial power rating $[\mathrm{MW}]$ is chosen to be the value when the cumulative density function of ramp rates distribution in Fig. 1 becomes the original SL. Fig. 5. shows the relationship between the initial power rating and the SL. The initial power rating for the given SL is calculated using the PDF of the Laplace distribution shown below

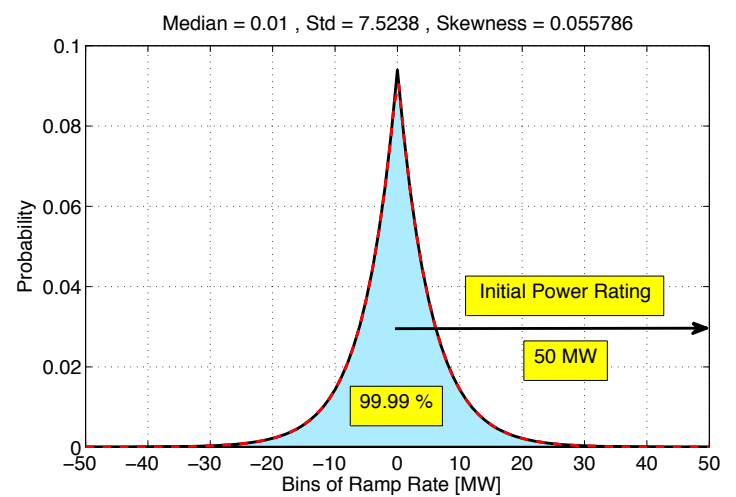

Fig. 5. Initial power rating and battery capacity can be estimated from the Laplace distribution. Initial power rating is the value when cumulative probability becomes the satisfaction level.

$$
\mathrm{SL}=2 \int_{0}^{\text {Power Rating }} \frac{1}{\sqrt{2} \sigma} \mathrm{e}^{-\frac{\sqrt{2} x}{\sigma}} d x
$$

and the power rating is given as

$$
\text { Power Rating }=-\frac{\sigma}{\sqrt{2}} \ln (1-\mathrm{SL})
$$

However, with a battery, the ramp rates of net production should be considered. The power rating in (7) will increase when ramp rates of the net production are considered. For example, if there are continuous ramp-up events that are violating the RRL, the power difference that a battery should compensate will also increase continuously. The battery power rating will depend on the relationship between the RRL and the characteristics of large ramps. Therefore, the power rating should depend on wind power data.

Third, the initial battery capacity is designed to be twice the power rating in MW per minute, because it is assumed that a battery must be able to charge or discharge energy corresponding to the power rating for a time interval at least one time when it is in the $0.5 \mathrm{SOC}$ level. Therefore, the initial battery capacity is given as

$$
\text { Battery Capacity }=\frac{\text { Power Rating }}{60} * 2
$$

Finally, the power rating is increased from the initial value while the compensated SL is being improved. At the same time, the power rating is also increased to follow the constraint in (9). If the compensated SL does not improve much, stop increasing the power rating but increasing the battery capacity continuously. After the compensated SL reaches to the target SL, battery parameters are slightly adjusted to find minimum values.

\section{Simulation \& Result}

This section provides three simulations of three methods of limiting ramp events. The results are compared to each other, and it shows that the battery operation based on the 
V.G. process has the best performance. The RRL is 30MW/Minute. The target SL is given as $99.99 \%$ in order to cover almost all severe ramp events. It should be noted that the original SL for wind power output is $74.13 \%$.

\section{A. Simulation}

The first simulation satisfies RRL using wind power curtailment and reserve requirements without the battery. This simulation assumes that the wind power is curtailed when it violates the upper RRL, and that the wind power is supported by the reserve services when it violates the lower RRL. The process of the first simulation is well shown in Fig. 6. During the ramp-up event from 30 to 50 min, the wind power is curtailed. On the contrary, during the severe ramp-down event from 70 to $140 \mathrm{~min}$, the wind power is supported by the reserve services. In the first simulation, the lost energy and required energy are calculated.

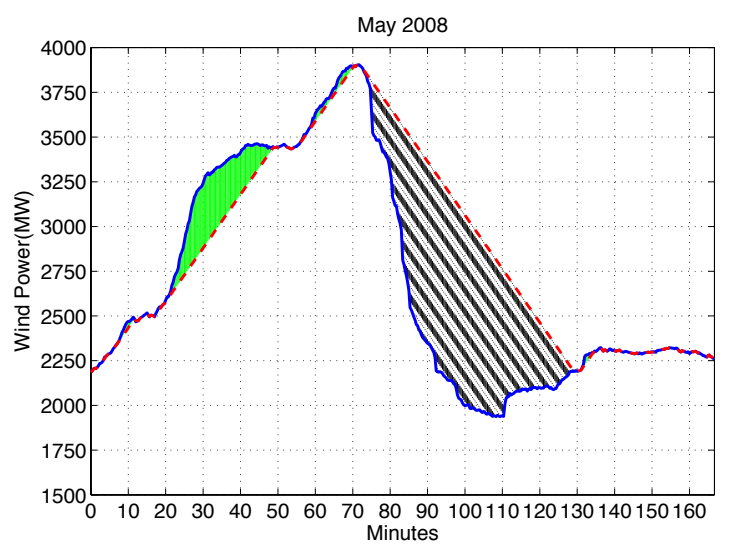

Fig. 6. The blue line represents the wind power, and the red dotted line represents the ramp-rate limited output. Lost energy is shown as a green line, and reserve energy is shown as a black dotted line.

The second simulation uses the basic battery algorithm only following the first strategy mentioned above without considering the V.G. process. Under the first strategy, the power rating and battery capacity are estimated empirically until it satisfies the SL. Finally, the third simulation uses the advanced battery algorithm following all three policies mentioned above based on the V.G. process. The battery parameters are also estimated.

\section{B. Result}

In the first simulation, the curtailed energy is $2,984 \mathrm{MWh}$, and the required energy is $2,724 \mathrm{MWh}$. Since the ramp rates of net production are limited by RRL, its SL becomes $100 \%$. In the second simulation, the power rating of the battery is set as 1,312 MW, and the storage capacity is set as 1,214 MWh. The result of the SOC is shown in Fig. 7. Since the second and third policies are not used, the SOC level does not prepare for future ramp events. Since the first strategy does not manage the SOC level, it will turn out that the battery capacity is bigger than the capacity in the third simulation, even though the compensated SL will be better in the third simulation.

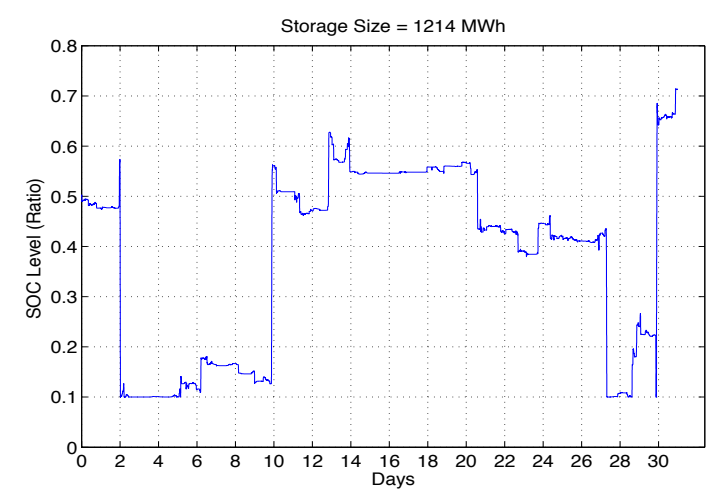

Fig. 7. The SOC level of the first strategy is only shown.

In the third simulation, the power rating is $1,110 \mathrm{MW}$, and the battery capacity is $652 \mathrm{MWh}$. The operation of the battery is shown in Fig. 8. The reason why battery operations are concentrated between RLLs is that the control strategy tries to keep the SOC around 0.5. without violating the RRL. When the net production does not violate the RLL, a battery can charge and discharge up to upper and lower RLLs by obeying the second policies.

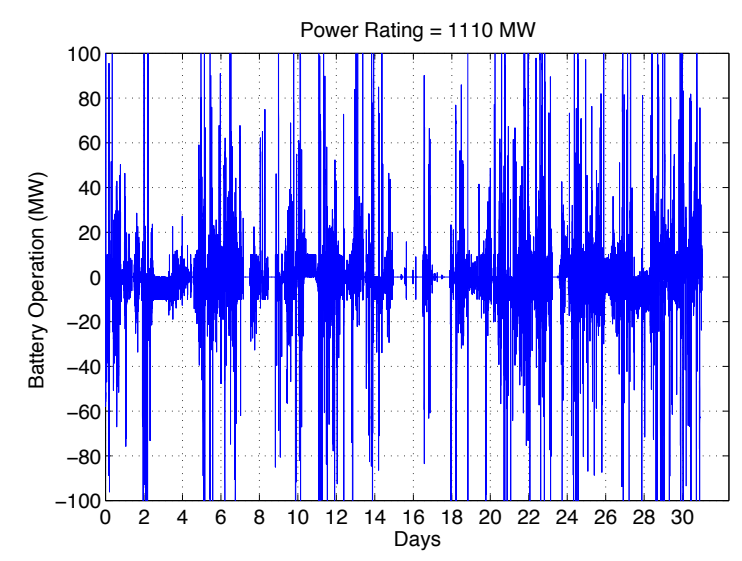

Fig. 8. The input and output of a battery operation

Fig. 9. shows the SOC level. The strategy tries to maintain an SOC of 0.5 to prepare for sudden and severe ramp events. The storage capacity of the third simulation is half of the capacity of the first simulation.

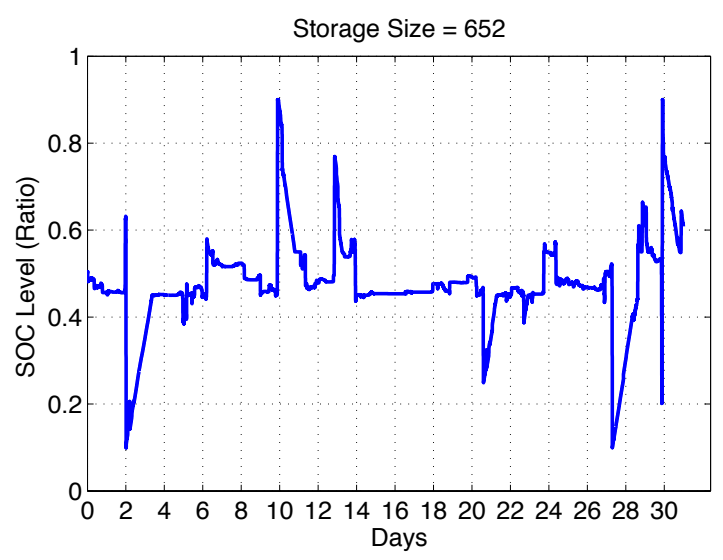

Fig. 9. The SOC level of $652 \mathrm{MWh}$ battery is shown. 
The results of the three simulations are summarized in Table II.

Table II. - Simulation Result

\begin{tabular}{|c|c|c|c|}
\hline & Curtailment & $\begin{array}{l}\text { Battery } \\
\text { Operation } \\
\text { (Without V.G. } \\
\text { process) }\end{array}$ & $\begin{array}{l}\text { Battery } \\
\text { Operation } \\
\text { (With } \\
\text { process) }\end{array}$ \\
\hline SL [\%] & $100 \%$ & 99.991 & 99.995 \\
\hline $\begin{array}{c}\text { Power Rating } \\
\text { [MW] }\end{array}$ & 1312 & 1110 \\
\hline $\begin{array}{c}\text { Battery } \\
\text { Capacity } \\
\text { [MWh] }\end{array}$ & 1214 & 652 \\
\hline $\begin{array}{c}\text { Energy Loss } \\
\text { [MWh] }\end{array}$ & 2984 & 535 & 416 \\
\hline $\begin{array}{c}\text { Reserve } \\
\text { [MWh] }\end{array}$ & 2724 & 0 & 0 \\
\hline
\end{tabular}

All three methods satisfied the target SL. Lost energy of both battery operation algorithms is much smaller than the lost and required energy of the first simulation. Table II. shows that considering the V.G. process can reduce the amount of battery capacity, so the V.G. process can reduce the cost of battery. However, since the power rating depends on the depth of ramp-down event or the height of ramp-up event, the power ratings for the second and third simulation are very similar. It is also clear that the power rating affects the compensated SL, but the changing battery capacity does not significantly affect the compensated SL. Therefore, we might reach the conclusion that a battery system requires a higher power rating, but it requires a relatively smaller battery capacity.

\section{Conclusion}

In this section, the summary and future works are enumerated.

\section{A. Summary}

The distribution of ramp rates of wind power output follows the symmetric Laplace distribution, ramp rates of non-overlapping ramp events are independent and have stationary increments, and the variances of ramp rates are linearly proportional to the duration of time interval over which the ramp is calculated for duration less than three minutes. Therefore, the V.G. process could be suggested to address the hidden uncertainty in deriving ramp rates of the wind power in a very short time. Based on the V.G. process, operational policies of a battery are suggested in order to limit severe ramp events. Furthermore, the power rating and battery capacity are also calculated.

Compared to the method using the curtailment and reserve services and the first strategy, the suggested operational strategy of using an algorithm based on the V.G. process avoids curtailment and the deployment of reserve to provide power. Furthermore, this simulation was conducted for total wind power output from all wind farms in ERCOT. Therefore, if a battery system will be installed in a single wind farm, smaller battery ratings will be enough.

\section{B. Future works}

Although this paper has focused on wind events, a similar approach could be used to limit ramps of Net load (load minus wind power). The results in this paper could be extended to the net load in order to estimate the reserve requirements for the ancillary services. Net load sampled every five minutes can be used to inform the dispatch signal in the ERCOT nodal market. Furthermore, shortterm weather forecasts, such as those forecasting a front, seasonal trend, diurnal trend should be used together in order to cope with ramp rates during a longer time. Finally, the Monte Carlo method can be used to test the suggested operating policies and to verify the battery parameters suggested using all three policies for more wind power scenarios.

\section{Acknowledgement}

This research was supported in part by the National Science Foundation under award ECCS-1065224. The authors thank to ERCOT for providing wind power data. Furthermore, the first author appreciates Xtreme Power (www.xtremepower .com) for supporting this research through an internship and providing base algorithms for the ramp rate control using the storage operation.

\section{References}

[1] R. A. Walling, "Analysis of Wind Generation Impact on ERCOT Ancillary Services Requirements", GE Energy, GE Project Team, March 2008

[2] N. Tanaka, "Harnessing Variable Renewables: A guide to the balancing challenge", March 2011, ISBN 978-92-6411138-7, International Energy Agency, Paris, France

[3] Teleke, S.; Baran, M.E.; Huang, A.Q.; Bhattacharya, S.; Anderson, L.; "Control Strategies for Battery Energy Storage for Wind Farm Dispatching," Energy Conversion, IEEE Transactions on, vol.24, no.3, pp.725-732, Sept. 2009

[4] E. Figueroa-Lopez, C. Houdre, “An overview of a nonparametric estimation method for Levy processes", Levy processes in Finance and Econometrics, Statistical and Applied Mathematical Sciences Institue (SAMSI), Oct. 2005

[5] S. Kotz, T. J. Kozubowski, K. Podgorski, "The Laplace Distribution and Generalizations: a revisit with applications to communications, economics, engineering, and finance", Birkhauser, Boston, 2001

[6] Madan, D. and E. Seneta. "The Variance Gamma (V.G.) Model for Share Market Returns." The Journal of Business Vol. 63, Issue. 4 (Oct 1990): 511-524,

[7] Louie, H.; "Evaluation of probabilistic models of wind plant power output characteristics," Probabilistic Methods Applied to Power Systems (PMAPS), 2010 IEEE 11th International Conference on, vol., no., pp.442-447, 14-17 June 2010, doi: 10.1109/PMAPS.2010.5528963

[8] Brekken, T.K.A.; Yokochi, A.; von Jouanne, A.; Yen, Z.Z.; Hapke, H.M.; Halamay, D.A.; "Optimal Energy Storage Sizing and Control for Wind Power Applications, "Sustainable Energy, IEEE Transactions on, vol.2, no.1, pp.69-77, Jan. 2011 